\begin{tabular}{|c|c|}
\hline Title & $\begin{array}{l}\text { Comparison of picosecond and nanosecond lasers for the synthesis of TiN sub-micrometer spherical particles by pulsed } \\
\text { laser melting in liquid }\end{array}$ \\
\hline Author(s) & $\begin{array}{l}\text { Sakaki, Shota; Saitow, Ken-ichi; Sakamoto, Masanori; Wada, Hiroyuki; Swiatkowska-W arkocka, Zaneta; Ishikawa, } \\
\text { Y oshie; Koshizaki, Naoto }\end{array}$ \\
\hline Citation & $\begin{array}{l}\text { A pplied Phy sics Express (A PEX), 11(3), } 035001 \\
\text { https://doi.org/10.7567/A PEX.11.035001 }\end{array}$ \\
\hline Issue Date & $2018-03$ \\
\hline Doc URL & http:/hdl.handle.net/2115/2703 \\
\hline Rights & (c) 2018 The Japan Society of A pplied Physics \\
\hline Type & article (author version) \\
\hline File Information & Picosecond_APEX_revision.pdf \\
\hline
\end{tabular}

Instructions for use 


\section{Comparison of picosecond and nanosecond lasers for the synthesis of TiN submicrometer spherical particles by pulsed laser melting in liquid}

Shota Sakaki ${ }^{1 *}$, Ken-ichi Saitow ${ }^{2,3}$, Masanori Sakamoto ${ }^{2}$, Hiroyuki Wada ${ }^{4}$, Zaneta Swiatkowska-Warkocka ${ }^{5}$, Yoshie Ishikawa ${ }^{6}$, and Naoto Koshizaki ${ }^{1 *}$

${ }^{1}$ Graduate School of Engineering, Hokkaido University, Sapporo, Hokkaido, 060-8628, Japan

${ }^{2}$ Department of Chemistry, Graduate School of Science, Hiroshima University, Higashi-hiroshima, Hiroshima, 739-8526, Japan

${ }^{3}$ Natural Science Center for Basic Research and Development (N-BARD), Hiroshima University, Higashi-hiroshima, Hiroshima, 739-8526, Japan

${ }^{4}$ School of Materials and Chemical Technology, Tokyo Institute of Technology, Yokohama, Kanagawa, 226-8502, Japan

${ }^{5}$ Institute of Nuclear Physics, Polish Academy of Sciences, PL-31342, Kraków, Poland

${ }^{6}$ Nanomaterials Research Institute, National Institute of Advanced Industrial Science and Technology (AIST), Tsukuba, Ibaraki, 305-8565, Japan

E-mail: sakaki.shota@frontier.hokudai.ac.jp, koshizaki.naoto@eng.hokudai.ac.jp

In this study, TiN submicrometer spherical particles were fabricated via pulsed laser melting in liquid using picosecond and nanosecond lasers applied to colloidal nanoparticles. The sizes of the obtained submicrometer spherical particles decreased as the pulse width decreased from nanoseconds to picoseconds. Further, the laser fluence required for fabricating submicrometer spherical particles by irradiation with a picosecond laser was lower than that with a nanosecond laser. This result suggests that the heat loss from the particles during pulsed laser heating is lower with shorter laser pulse durations. Therefore, picosecond laser irradiation is an energy-efficient method for fabricating submicrometer spherical particles. 
Nanoparticle synthesis via irradiation with a high-power laser in a liquid medium, which is known as pulsed laser ablation in liquid (PLAL), has been studied for a few decades because of the distinctive particle formation mechanism associated with this technique. ${ }^{1-5)}$ This method can be applied to various materials such as metals, ${ }^{6)}$ oxides, ${ }^{7)}$ carbides, ${ }^{8)}$ and alloys ${ }^{9)}$. In PLAL, high-power pulsed lasers (nanosecond, ${ }^{8)}$ picosecond, ${ }^{10)}$ and femtosecond lasers ${ }^{10)}$ ) have been widely used because they can provide the high instantaneous energy density needed to elicit an explosive interaction between a target and laser light. For excitation of a target with ultrashort pulses such as femtosecond laser, intensive heating of an electron gas occurs during the pulse duration because pulse duration is shorter than electron-phonon coupling period. ${ }^{11)}$ In contrast, for excitation of a target with nanosecond pulses, the electrons and lattice are in thermal equilibrium because pulse duration is longer than electron-phonon coupling period. ${ }^{11)}$

Recently, submicrometer spherical particle formation by pulsed laser melting in liquid (PLML) has been reported. ${ }^{5,12)}$ In PLML, nanosecond laser is usually used for synthesis of submicrometer spherical particles, since PLML is thermal process under equilibrium conditions between electrons and lattice. ${ }^{13-15)}$ Heat loss during nanosecond heating has been considered to be negligible because a typical time needed for particle cooling is much larger than duration of nanosecond laser heating. ${ }^{16)}$ However, recent study indicated that the particles dissipate heat energy by cooling effect of surrounding liquid even during several tens of nanoseconds heating. ${ }^{17)}$ Therefore, laser irradiation with shorter pulse width than nanoseconds would be effective in reducing heat loss of pulsed heating.

Barcikowski et al. reported that submicrometer spherical particles could be synthesized by picosecond laser irradiation to colloidal particles. ${ }^{18,19)}$ This method was effective when the pulse width was longer than the electron-phonon coupling period of the material. ${ }^{18)}$ However, the difference between picosecond and nanosecond laser melting in liquid has not been investigated. Thus, in this study, submicrometer spherical particles were fabricated via both picosecond and nanosecond laser irradiation under similar conditions to study the effect of picosecond laser irradiation on the energy efficiency of particle synthesis and the properties of the obtained particles. TiN was used as the materials for the particles in this study because it has potential applications as submicrometer hard plasmonic particles. ${ }^{20,21)}$

TiN raw particles (Wako Pure Chemical Industries, Ltd., < $50 \mathrm{~nm}$ ) were dispersed in 
deionized water at a concentration of $0.2 \mathrm{~g} / \mathrm{L}$. A nanosecond laser (Continuum Powerlite Precision 8000, $532 \mathrm{~nm}$ wavelength, $10 \mathrm{~Hz}$ pulse frequency, $7 \mathrm{~ns}$ pulse width, and $8 \mathrm{~mm}$ beam diameter) and a picosecond laser (Continuum PY61C-10, $532 \mathrm{~nm}$ wavelength, $10 \mathrm{~Hz}$ pulse frequency, 40 ps pulse width, and $6 \mathrm{~mm}$ beam diameter) were used for laser irradiation. A colloidal solution of TiN raw particles was agitated using a magnetic stirrer during laser irradiation. To obtain sufficient laser fluence for PLML, the laser beam of the picosecond laser was condensed to a $4 \mathrm{~mm}$ diameter through a convex lens with a focal length of 500 $\mathrm{mm}$. To compensate for the difference in the sizes of the laser beams (the beam of the nanosecond laser was four times larger than that of the picosecond laser), $3 \mathrm{ml}$ of suspension were irradiated for 20 min with the picosecond laser and $6 \mathrm{ml}$ of the suspension were irradiated for 10 min with the nanosecond laser (total input energy per suspension volume irradiated with the picosecond laser was equal to that with the nanosecond laser). The sizes and morphologies of the particles were measured via field-emission scanning electron microscopy (FE-SEM JEOL JSM-6500F). The crystal structures of particles were evaluated by X-ray diffraction analysis (XRD, Rigaku RINT-2000).

SEM images of particles irradiated with a nanosecond laser for $10 \mathrm{~min}$ are shown in Figs. 1(a) and 1(b). Nanosecond laser irradiation at a laser fluence of $67 \mathrm{~mJ}$ pulse $^{-1} \mathrm{~cm}^{-2}$ resulted in submicrometer spherical particles; in contrast, irradiation at a laser fluence of $50 \mathrm{~mJ}$ pulse $^{-1} \mathrm{~cm}^{-2}$, the obtained particles were almost identical to the raw particles. Figures 1(c) and 1(d) show the particles irradiated with a picosecond laser for $20 \mathrm{~min}$. Picosecond laser irradiation at a laser fluence of 50 and $67 \mathrm{~mJ} \mathrm{pulse}^{-1} \mathrm{~cm}^{-2}$ resulted in submicrometer spherical particle formation. Thus, a low laser fluence is sufficient to synthesize submicrometer spherical particles with a small pulse width. This result indicates that the heat loss from particles irradiated with a picosecond laser is lower than that from particles irradiated with a nanosecond laser during pulsed laser heating.

Figures 2(a) and 2(c) shows SEM images of particles irradiated with a pulsed laser with different pulse widths at a laser fluence of $67 \mathrm{~mJ}_{\text {pulse }} \mathrm{cm}^{-2}$. The final diameter of particle irradiated with a pulsed laser for sufficient time is determined by the laser fluence, because the laser fluence determines the maximum diameter of the particle which can be melted. ${ }^{22}$ To irradiate with a pulsed laser for sufficient time, the irradiation times were three times longer than those used on the particles shown in Fig. 1; the irradiation time with the 
nanosecond laser was $30 \mathrm{~min}$ and that with the picosecond laser was $60 \mathrm{~min}$. Based on these SEM images, the size distributions of the submicrometer spherical particles irradiated with a pulsed laser with different pulse widths were graphed by measuring the sizes of more than 300 particles [Figs. 2(b) and 2(d)]. The average diameter of the particles irradiated with the nanosecond laser was $111 \mathrm{~nm}$ and that of the particles irradiated with the picosecond laser was $74 \mathrm{~nm}$. This finding indicates that the average diameter of submicrometer spherical particles also decreases with decreasing pulse width.

Figure 3 shows XRD patterns of the particles depicted in Fig. 2, which were irradiated with picosecond and nanosecond lasers at a laser fluence of $67 \mathrm{~mJ}$ pulse $^{-1} \mathrm{~cm}^{-2}$; a logarithmic ordinate scale was used to emphasize peaks from minor components of particles. Both samples exhibited strong XRD peaks corresponding to TiN. For nanosecond laser irradiation, peaks corresponding to rutile $\mathrm{TiO}_{2}$ appeared, indicating a slight oxidation of TiN. This result is consistent with a previous report of the formation of $\mathrm{TiO}_{2}$ and $\mathrm{TiO}_{\mathrm{x}} \mathrm{N}_{\mathrm{y}}$ by PLML. ${ }^{23)}$ In contrast, for picosecond laser irradiation, there was no peak indicating the presence of rutile $\mathrm{TiO}_{2}$, but the broad and weak peaks in the XRD diffraction pattern corresponding to $\mathrm{TiO}_{\mathrm{x}} \mathrm{N}_{\mathrm{y}}$ were observed in higher $2 \theta$ angles relative to the peaks corresponding to $\mathrm{TiN}^{24)}$ The particle heating time with a picosecond laser is shorter than that with a nanosecond laser, and the cooling time of the small particles obtained by picosecond laser irradiation is shorter than that of the large particles obtained by nanosecond laser irradiation. ${ }^{16,17)}$ Therefore, the oxidation reaction between particles and liquid may be suppressed due to the reduction in reaction time at high temperature.

The thermal diffusion length during pulsed laser heating is given by the following well-known diffusion equation:

$$
l(t)=\sqrt{\frac{2 \cdot k \cdot \tau}{\rho \cdot C}},
$$

where $k$ is the heat conductivity of the particles, $\tau$ is the pulse width, $\rho$ is the density of the particles, and $C$ is the specific heat of the particles. Thermal diffusion length in TiN particles during pulsed laser heating calculated by Eq. (1) based on the physical properties of $\mathrm{TiN}^{25)}$ in Table I is $17.6 \mathrm{~nm}$ for picosecond laser and $233 \mathrm{~nm}$ for nanosecond laser. The thermal diffusion length increases with the heating duration.

Figure 4 shows the spatial distribution of the resistive heat loss density from TiN 
spherical particles of $50 \mathrm{~nm}$ and $100 \mathrm{~nm}$ in diameter owing to electromagnetic wave irradiation by a plane-polarized light with a wavelength of $532 \mathrm{~nm}$ using the constants in Table II. The distributions were calculated using COMSOL Multiphysics ${ }^{\circledR}$, a commercial software based on the finite element method. In this size range, TiN particles are heated mainly at their surfaces by the laser light irradiation. The thermal diffusion length during nanosecond laser heating $(233 \mathrm{~nm})$ is larger than the diameter of the particles obtained by nanosecond laser irradiation. Therefore, thermal energy diffuses from the particle surface which absorbs laser energy to the entire particle during pulsed laser heating, resulting in homogeneous heating and melting the particles. In contrast, the thermal diffusion length during picosecond laser heating $(17.6 \mathrm{~nm})$ is smaller than the diameter of the particles obtained by picosecond laser irradiation. Therefore, particles are partially heated at the incident light side of the particle surface during pulsed laser heating. The size of the obtained particles decreases with decreasing thermal diffusion length, which depends on the pulse width.

As shown experimentally and theoretically, the particle heating-cooling model developed by our group can be applied to calculate the particle temperature during pulsed laser irradiation. ${ }^{17)}$ Particles are heated via laser absorption depending on the time profile of laser pulse. The absorption efficiency can be calculated from the refractive index and extinction coefficient of $\mathrm{TiN}^{26)}$ shown in Table II based on the Mie theory. In this model, the heat loss due to particle cooling is considered, and it is assumed that all laser energy absorbed by the particles is converted into heat energy. Hence, the laser fluence required for the melting start are calculated based on this particle heating-cooling model considering the cooling effect of the surrounding liquid.

The fluence-size phase diagram for TiN spherical particles determined by the previously reported procedure is shown in Fig. $5 .{ }^{16,17)}$ The phase diagram shows the phase boundary curves for the melting start when particles are irradiated with a nanosecond laser (red dotted line), a picosecond laser (blue solid line), and a pulsed laser without heat loss (black dashed line). With nanosecond laser heating, a higher amount of laser energy is needed to start the melting than under conditions without heat loss, especially with small particles $(<50 \mathrm{~nm})$. Small particles are drastically cooled by the surrounding liquid because the cooling effect depends on the heat transfer coefficient, which is inversely proportional to the particle 
diameter. ${ }^{17)}$ With picosecond laser heating, however, the laser energy required to start the melting is almost the same as that without heat loss; almost all of the laser energy is transferred for particle heating because the heating rate is much faster than the cooling rate. Therefore, picosecond laser heating is an energy-efficient process for synthesizing submicrometer spherical particles.

This phase diagram illustrates that nanosecond laser irradiation at a laser fluence of 50 $\mathrm{mJ}$ pulse $\mathrm{cm}^{-1}$ is insufficient to melt the particles owing to the particle cooling effect. Therefore, submicrometer spherical particles could not be synthesized via nanosecond laser irradiation with a laser fluence of $50 \mathrm{~mJ}$ pulse $\mathrm{cm}^{-2}$. By increasing the laser fluence, the size of the particles produced via nanosecond PLML is reported to be increased. ${ }^{14-17)}$ This is probably due to the repetitive melting and fusing process of the particles. ${ }^{22)}$ In PLML, the agglomerates of the particles melt and fuse, resulting in relatively large particles formation. Then, the growth process continues as long as the particles can be melted. The phase diagram shown in Fig. 5 suggests that at a laser fluence of $67 \mathrm{~mJ}$ pulse $\mathrm{cm}^{-2}$, particles smaller than $120 \mathrm{~nm}$ melt to form submicrometer spherical particles; the particles experimentally obtained by nanosecond laser irradiation are approximately $111 \mathrm{~nm}$ in size, as measured from the SEM images. However, the particles obtained by picosecond laser irradiation were much smaller than the size estimated from the fluence-size phase diagram and were close to the raw particle size. This is probably due to insufficient fusing induced by the inhomogeneous heating of raw particles by picosecond laser heating.

Figure 6 shows an SEM image of particles irradiated with a picosecond laser for $20 \mathrm{~min}$ at a laser fluence of $167 \mathrm{~mJ}$ pulse $\mathrm{cm}^{-2}$. Nanoparticles were mainly observed, but a few submicrometer spherical particles were seen. In case of nanosecond laser heating, nanoparticles were main product at a laser fluence of $350 \mathrm{~mJ}$ pulse ${ }^{-1} \mathrm{~cm}^{-2}$. ${ }^{23}$ ) The laser fluence at which nanoparticles were mainly formed decreased with decreasing pulse width. The laser intensity ( $I=J / \tau$ where $J$ is the laser fluence and $\tau$ is pulse width) of the picosecond (40 ps) laser exceeds $10^{13} \mathrm{~W} / \mathrm{m}^{2}$, which is two orders of magnitude larger than that of nanosecond (7 ns) laser. The thermal diffusion length during picosecond laser heating is smaller than the size of the agglomerates. Therefore, the agglomerates of raw particles partially evaporate under high-intensity picosecond laser irradiation, resulting in nanoparticle formation. The Coulomb explosion of the particles would not occur because the 
electron-phonon coupling period (10 fs for TiN at $298 \mathrm{~K}$ ) is shorter than the picosecond time scale. $^{27)}$

Because the laser fluence threshold for submicrometer spherical particle formation decreased as the pulse width decreased (from nanoseconds to picoseconds), it can be concluded that the heat loss during pulsed laser melting in liquid is reduced in the case of ultrafast heating. The calculations also show that particles are heated with negligible heat loss during the pulsed laser heating with a picosecond laser. Therefore, the picosecond laser irradiation of colloidal particles is an energy-efficient method for pulsed laser melting in liquid. The oxidation reaction is suppressed by picosecond laser irradiation because the reaction time at the high temperature induced by picosecond laser irradiation is shorter than that with nanosecond laser irradiation. The size of the obtained particles also decreased with decreasing pulse width from nanoseconds to picoseconds, which can be attributed to the decrease in the thermal diffusion length in the particles. The thermal diffusion length during picosecond laser heating is smaller than the size of the particles, implying that the particles irradiated with a picosecond laser are only partially heated. This result suggests that tuning the thermal diffusion length by varying the pulse width may lead to size control of submicrometer spherical particles.

\section{Acknowledgments}

This work was supported by JSPS KAKENHI Grant Numbers 26289266 and 26870908. 
Template for APEX (Jan. 2014)

\section{References}

1) C.-Y. Shih, C. Wu, M. V. Shugaev, and L. V. Zhigilei, J. Colloid Interface Sci. 489, 3 (2017).

2) S. Ibrahimkutty, P. Wagener, T. d. S. Rolo, D. Karpov, A. Menzel, T. Baumbach, S. Barcikowski, and A. Plech, Sci. Rep. 5, 16313 (2015).

3) K. Sasaki, T. Nakano, W. Soliman, and N. Takada, Appl. Phys. Exp. 2, 046501 (2009).

4) S. Ibrahimkutty, P. Wagener, A. Menzel, A. Plech, and S. Barcikowski, Appl. Phys. Lett. 101, 103104 (2012).

5) D. Zhang, B. Gökce, and S. Barcikowski, Chem. Rev. 117, 3990 (2017).

6) F. Mafuné, J.-y. Kohno, Y. Takeda, T. Kondow, and H. Sawabe, J. Phys. Chem. C 104, $9111(2000)$.

7) N. N. Luo, X. Tian, C. Yang, J. Xiao, W. Hu, D. Chen, and L. Li, Phys. Chem. Chem. Phys. 15, 12235 (2013).

8) V. Amendola, P. Riello, and M. Meneghetti, J. Phys. Chem. C 115, 5140 (2011).

9) Y. Ishikawa, K. Kawaguchi, Y. Shimizu, T. Sasaki, and N. Koshizaki, Chem. Phys. Lett. 428, 426 (2006).

10) S. Barcikowski, A. Menéndez-Manjón, B. Chichkov, M. Brikas, and G. Račiukaitis, Appl. Phys. Lett. 91, 083113 (2007).

11) S.-S. Wellershoff, J. Hohlfeld, J. Güdde, and E. Matthias, Appl. Phys. A 69, 99 (1999).

12) Y. Ishikawa, Y. Shimizu, T. Sasaki, and N. Koshizaki, Appl. Phys. Lett. 91, 161110 (2007).

13) H. Wang, M. Miyauchi, Y. Ishikawa, A. Pyatenko, N. Koshizaki, Y. Li, L. Li, X. Li, Y. Bando, and D. Golberg, J. Am. Chem. Soc. 133, 19102 (2011).

14) H. Wang, N. Koshizaki, L. Li, L. Jia, K. Kawaguchi, X. Li, A. Pyatenko, Z. Swiatkowska-Warkocka, Y. Bando, and D. Golberg, Adv. Mater. 23, 1865 (2011).

15) H. Wang, A. Pyatenko, K. Kawaguchi, X. Li, Z. Swiatkowska-Warkocka, and N. Koshizaki, Angew. Chem. Int. Ed. 49, 6361 (2010).

16) A. Pyatenko, H. Wang, N. Koshizaki, and T. Tsuji, Laser Photonics Rev. 7, 596 (2013).

17) S. Sakaki, H. Ikenoue, T. Tsuji, Y. Ishikawa, and N. Koshizaki, ChemPhysChem 18, 1101 (2017).

18) D. Zhang, M. Lau, S. Lu, S. Barcikowski, and B. Gökce, Sci. Rep. 7, 40355 (2017).

19) M. Lau, and S. Barcikowski, Appl. Surf. Sci. 348, 22 (2015).

20) S. Ishii, R. P. Sugavaneshwar, and T. Nagao, J. Phys. Chem. C 120, 2343 (2016).

21) G. V. Naik, J. L. Schroeder, X. Ni, A. V. Kildishev, T. D. Sands, and A. Boltasseva, Opt. Mater. Express 2, 478 (2012).

22) A. Pyatenko, H. Wang, and Naoto Koshizaki, J. Phys. Chem. C 118, 4495 (2014).

23) K. Kawasoe, Y. Ishikawa, N. Koshizaki, T. Yano, O. Odawara, and H. Wada, Appl. Phys. B 119, 475 (2015).

24) N. Martin, O. Banakh, A.M.E. Santo, S. Springer, R. Sanjinés, J. Takadoum, and F. Lévy, Appl. Surf. Sci. 185, 123 (2001).

25) S. Jeon, C. J. V. Tyne, and H. Lee, Ceram. Int. 40, 8677 (2014).

26) P. Patsalas, N. Kalfagiannis, and S. Kassavetis, Materials 8, 3128 (2015).

27) A. B. Mei, A. Rockett, L. Hultman, I. Petrov, and J. E. Greene, J. Appl. Phys. 114, 193708 (2013) 


\section{Figure Captions}

Fig. 1. SEM images of TiN particles irradiated (a, b) with a nanosecond laser for $10 \mathrm{~min}$ and (c, d) with a picosecond laser for 20 min with laser fluence values of (a, c) $50 \mathrm{~mJ}$ pulse ${ }^{-1} \mathrm{~cm}^{-2}$ and (b, d) $67 \mathrm{~mJ}$ pulse $\mathrm{cm}^{-1}$.

Fig. 2. SEM images of TiN particles irradiated (a) with a nanosecond laser for $30 \mathrm{~min}$ and (c) with a picosecond laser for $60 \mathrm{~min}$ at the laser fluence of $67 \mathrm{~mJ} \mathrm{pulse}^{-1} \mathrm{~cm}^{-2}$ and the corresponding size distributions of TiN particles irradiated (b) with a nanosecond laser for $30 \mathrm{~min}$ and (d) with a picosecond laser for $60 \mathrm{~min}$.

Fig. 3. XRD patterns of the particles irradiated with a picosecond and nanosecond lasers at a laser fluence of $67 \mathrm{~mJ}$ pulse $\mathrm{cm}^{-2}$ depicted in Fig. 2 .

Fig. 4. Spatial distribution of density of the resistive heat loss from TiN spherical particles with diameters of (a) $50 \mathrm{~nm}$ and (b) $100 \mathrm{~nm}$ when excited by plane-polarized light with a wavelength of $532 \mathrm{~nm}$, resulting in local thermal heating by the electromagnetic wave (light) irradiation. Here, the light propagates in the X-direction, the electric field oscillates in the $\mathrm{X}-\mathrm{Z}$ plane, and the magnetic field oscillates in the $\mathrm{X}-\mathrm{Y}$ plane. The distributions were calculated using COMSOL Multiphysics ${ }^{\circledR}$.

Fig. 5. Phase diagram of TiN spherical particles at the melting start calculated according to the particle heating-cooling model and accounting for the cooling effect of the surrounding liquid. Irradiation with a nanosecond laser (red dotted line), a picosecond laser (blue line), and a laser without heat loss (black dashed line) are shown. The average diameters of the obtained particles after nanosecond laser irradiation (red circle) and picosecond laser irradiation (blue triangle) are also plotted.

Fig. 6. SEM image of TiN particles irradiated with a picosecond laser for 20 min with a laser fluence of $167 \mathrm{~mJ}$ pulse $\mathrm{cm}^{-2}$ 
Template for APEX (Jan. 2014)

Table I. Physical properties of TiN

\begin{tabular}{ccc}
$\rho$ & & \\
$\mathrm{g} / \mathrm{cm}^{3}$ & & \\
) & $k(\mathrm{~W} / \mathrm{m} \cdot \mathrm{K})$ & $C(\mathrm{~kJ} / \mathrm{kg} \cdot \mathrm{K})$ \\
\hline 5.22 & 19.2 & 0.951 \\
\hline
\end{tabular}

Table II. Optical properties of TiN (532 nm)

\begin{tabular}{cc} 
refractive index & extinction coefficient \\
\hline 1.42 & 1.48 \\
\hline
\end{tabular}


Template for APEX (Jan. 2014)

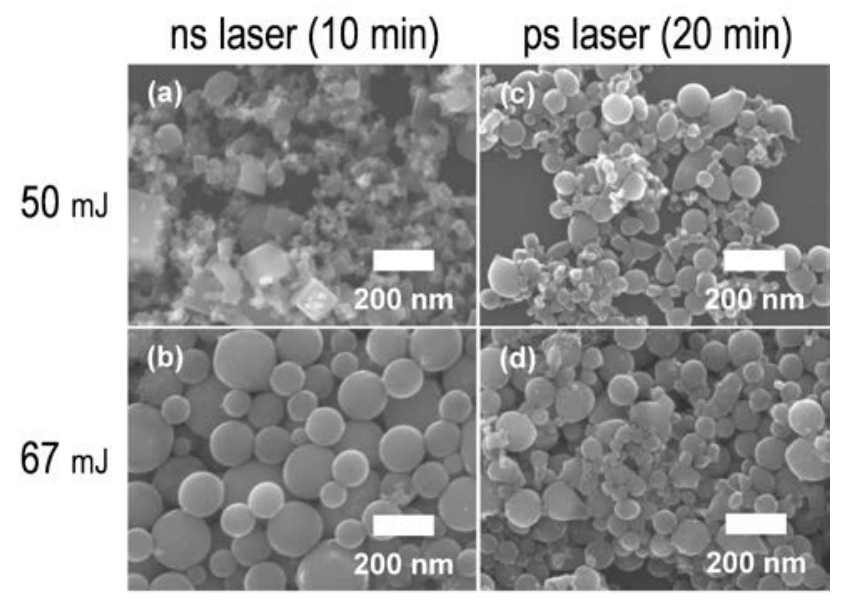

Fig.1.

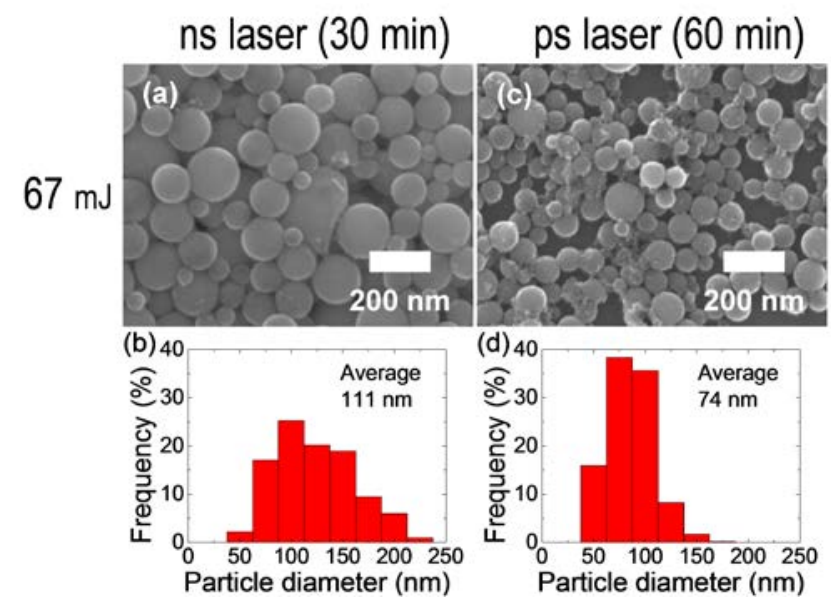

Fig. 2. 
Template for APEX (Jan. 2014)

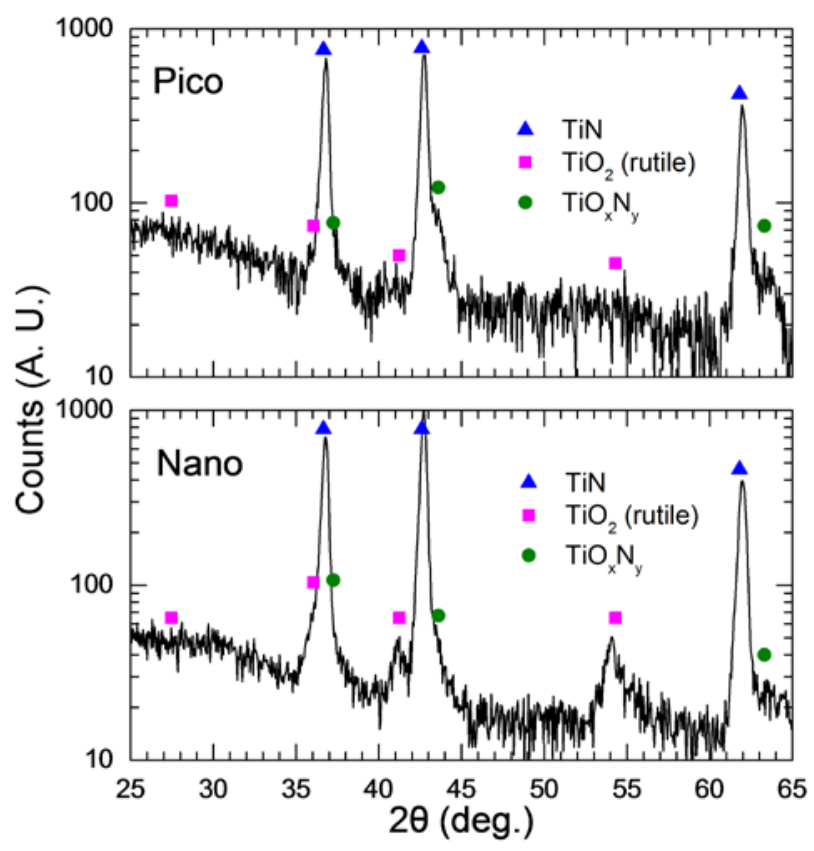

Fig. 3.

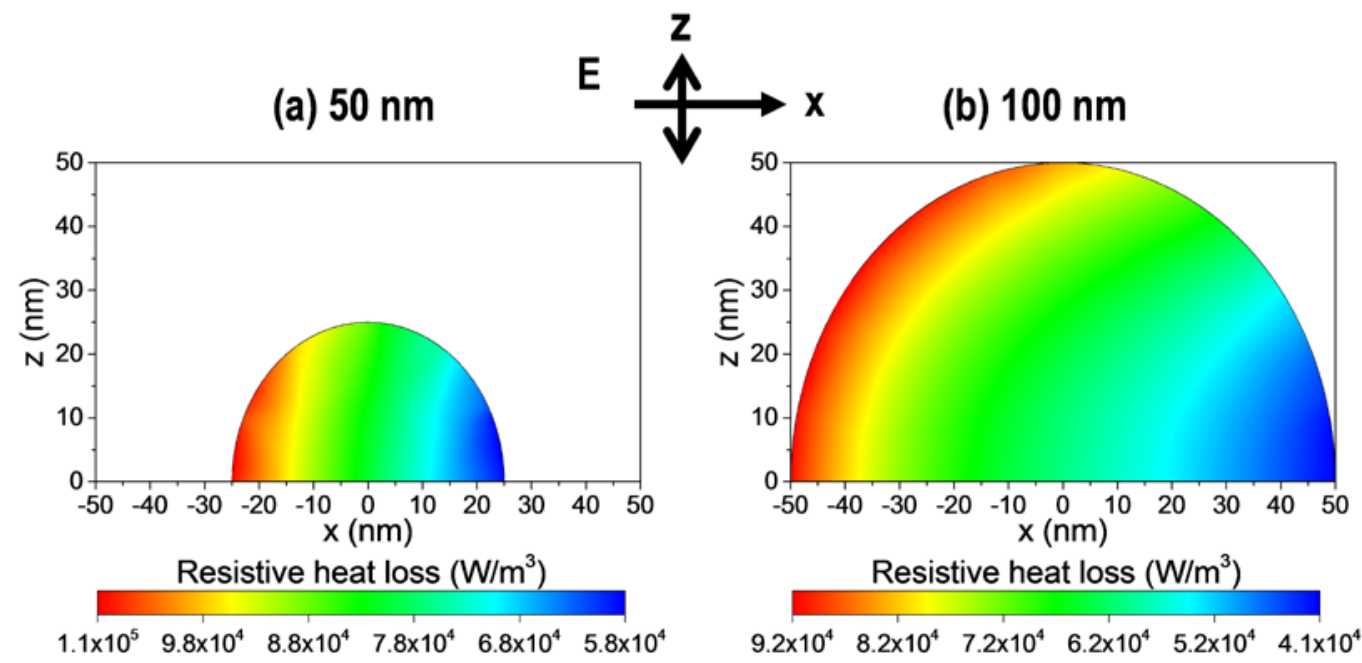

Fig. 4. 
Template for APEX (Jan. 2014)

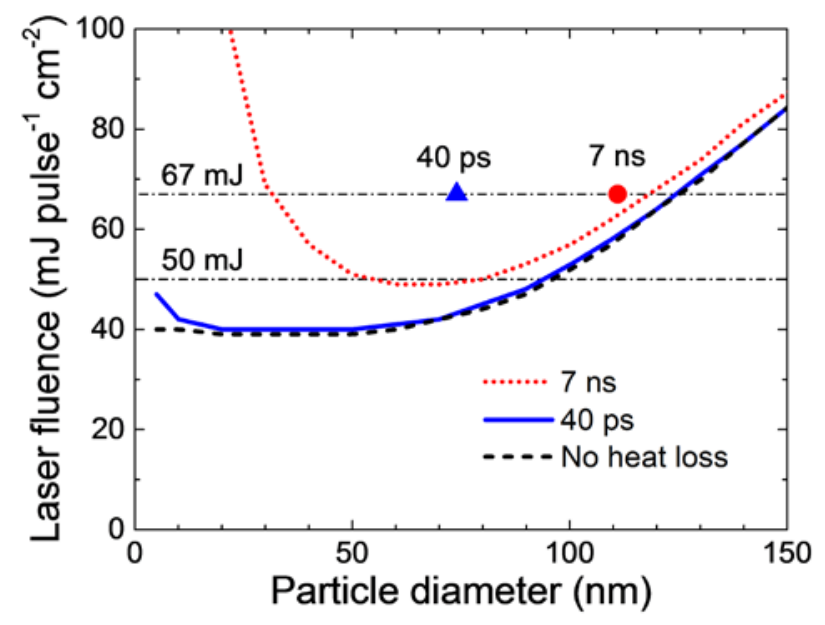

Fig. 5.

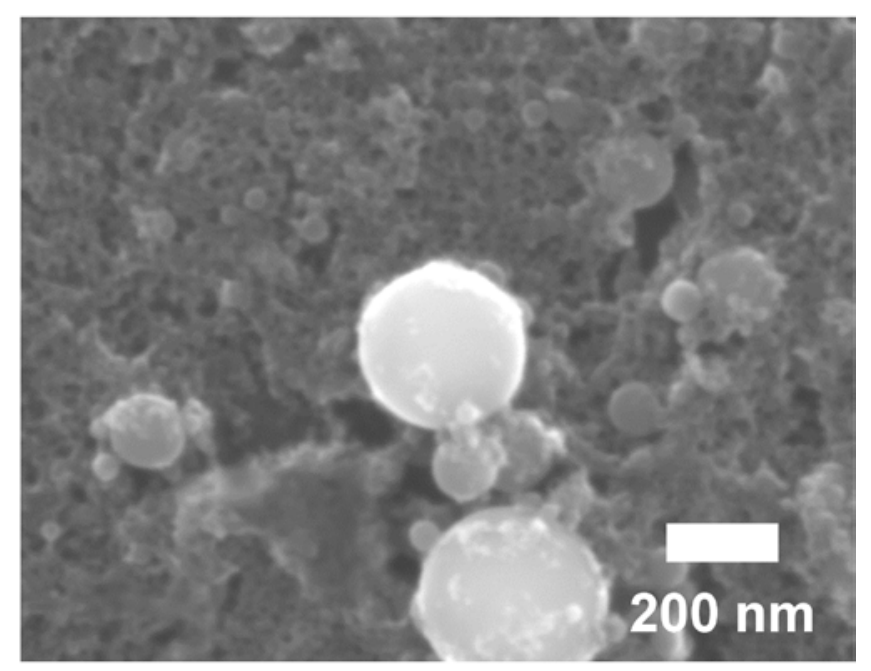

Fig. 6. 